\title{
Horkheimer, lector del positivismo. Un análisis crítico de la interpretación horkheimeriana del positivismo en sus textos tempranos
}

\section{[Horkheimer's Reading of Positivism. A Critical Analysis of Horkheimer's Interpretation of Positivism in His Early Writings]}

\author{
ADRIANA GONZALO \\ Instituto de Investigaciones en Humanidades y Ciencias Sociales \\ Consejo Nacional de Investigaciones Científicas y Técnicas \\ Universidad Nacional del Litoral \\ adriana.n.gonzalo@gmail.com \\ PAULA GARCÍA CHEREP \\ Instituto de Investigaciones en Humanidades y Ciencias Sociales \\ Consejo Nacional de Investigaciones Científicas y Técnicas \\ Universidad Nacional del Litoral \\ paulagcherep@yahoo.com
}

Resumen: Este trabajo reconstruye la caracterización de "positivismo" en tres obras centrales de Horkheimer. Se afirma que, en el uso del término, Horkheimer incurre en una doble equivocidad, pues lo utiliza indistintamente para referirse al positivismo decimonónico y al del siglo xx; además, no reconoce las diferencias internas entre las posiciones de los positivistas contemporáneos. Asimismo, se muestra que muchos rasgos que se consignan como propios del positivismo del siglo XX no tienen justificación en los textos de esa tradición, de manera que la categoría horkheimeriana de "positivismo" se convierte en una entidad interpretativa respecto de la cual la Escuela de Fráncfort se considera antagónica. A partir de lo anterior se realiza una crítica a las interpretaciones del autor.

Palabras clave: lectura crítica, Escuela de Fráncfort, metafísica, teoría científica, empirismo lógico

\begin{abstract}
This paper reconstructs the characterization of "positivism" in Horkheimer's works. We argue that in the use of this term, Horkheimer makes a misinterpretation, then he uses it in order to refer to both nineteenth century positivism and the positivism of the twentieth century; at the same time, he does not recognize the significant contrasts between the positions of the twentieth century positivists. We also show that many of the features Horkheimer sets out for twentieth century positivists find no correlation in texts belonging to this tradition, which makes Horkheimer's concept of "positivism" an interpretative entity in opposition to Frankfurt School. From the foregoing, we carry out a critique of the interpretations made by the author. Key words: critical reading, Frankfurt School, metaphysics, scientific theory, logical empiricism
\end{abstract}




\section{Introducción}

El presente artículo sigue la línea de revisión histórica del positivismo lógico ${ }^{1}$ y está enmarcado en una investigación acerca de su recepción e interpretación en la tradición de la Escuela de Fráncfort. En las siguientes páginas examinaremos críticamente la recepción del positivismo en tres obras tempranas de Horkheimer.

A partir del análisis de un corpus conformado por tres obras (Horkheimer 2015 [1931], Horkheimer 2000 [1937], Horkheimer 2002 [1937]), destacaremos los puntos centrales de la caracterización de "positivismo" o expresiones afines en ellas. Al respecto, conviene resaltar que el término designa muchas veces tanto (a) al positivismo decimonónico, como (b) al positivismo del siglo xx. ${ }^{2}$ También hacemos notar que en muchos casos "positivismo" se refiere a (c) una entidad interpretativa respecto de la cual la posición de los miembros de la Escuela de Fráncfort se considera antagónica.

Después, mostramos que, en general, no se visualizan diferencias interpretativas entre el positivismo en los sentidos (a) y (b) y las que se asignan al (b) son una extensión de rasgos visibles solamente en las posiciones del positivismo en el sentido (a). Además, mostramos que la caracterización del positivismo (b) se realiza desde una concepción uniformista del movimiento y que no se reconocen las diferencias entre los autores representativos del positivismo del siglo Xx. Por último, mostraremos que muchos de los rasgos caracterizadores del positivismo (b) no tienen un correlato justificante en los textos de los autores pertenecientes a la tradición y constituyen una caracterización del positivismo en el sentido (c).

A partir de lo anterior haremos una crítica a las interpretaciones realizadas por el autor para mostrar que resultan sesgadas parcialmente por algunos prejuicios que conducen a la constitución de (c). Resaltaremos que la interpretación que Horkheimer hace del positivismo o bien desconoce una buena parte de la producción de sus autores, o bien in-

${ }^{1}$ Cfr. Cirera, Ibarra y Mormann 1996; Coffa 1991; Friedman 1999; Giere y Richardson 1996; Ibarra y Mormann 2003; Mormann 2011; Parrini et al. 2003; Reisch 2009; Richardson y Uebel 2007; Stadler 2003; Stadler 2010; Uebel 1992; Uebel 1993; Uebel 1996a; Uebel 1996b; Uebel 2005; Uebel 2009 y Uebel 2010.

${ }^{2}$ Consideraremos aquí la expresión "positivismo del siglo xx" equivalente a "positivismo lógico", "neopositivismo" o "empirismo lógico", a pesar de que no haya unanimidad en las equivalencias extensionales de estas denominaciones. En seguida, restringiremos tal denominación sobre todo al Círculo de Viena. Como es sabido, la etiqueta suele extenderse al Círculo de Berlín y a otros movimientos filosóficos posteriores.

Revista de Filosofía Diánoia, vol. 64, no. 83 (noviembre de 2019-abril de 2020) e-ISSN: 1870-4913 • DOI: https://doi.org/10.22201/iifs.18704913e.2019.83.1717 
terpreta esta última con el único fin de cuestionarla, para luego posibilitar la constitución de la identidad propia de la posición de la Escuela de Fráncfort.

Nos proponemos mostrar que la estrategia central de Horkheimer consiste en pasar de una caracterización epistemológica del positivismo a una valoración práctico-política que señala los límites y desaciertos del movimiento, de manera que se contrapone a la concepción francfortiana, que resulta una posición superadora de aquél.

\section{La construcción interpretativa del "positivismo" en Horkheimer}

2.1. Análisis de la recepción del positivismo en Horkheimer 2015 [1931]

En su conferencia de 1931, Horkheimer sostiene que la situación de la investigación social está marcada en ese momento por una separación entre la filosofía y lo que concibe como la "ciencia positiva" de la sociedad. El autor entiende que el positivismo representa las tendencias individualistas de la sociedad capitalista propias del modelo europeo contemporáneo, que bregan por una estrecha relación entre ciencia, técnica y producción industrial con vistas a un ideal de progreso lineal e indefinido.

En oposición a esa visión, Horkheimer estima que la recuperación de las bases del sistema hegeliano puede dar fundamento a la nueva filosofía social, enriquecida con los aportes de las ciencias empíricas, pero sin dejar de lado la pregunta por las esencias ni la comprensión de la subjetividad en el proceso de constitución del saber. En este sentido, afirma que "la filosofía social [...] ha sido llamada con urgencia para cumplir de una forma renovada esa función que le fue asignada por Hegel. Y la filosofía social ha escuchado este llamado" (Horkheimer 2015 [1931], p. 216).

Desde el punto de vista de Horkheimer, el positivismo propone una indagación fragmentada que sólo ve los hechos desde una actitud naturalista y cuyo análisis no puede ir más allá de ellos. En este sentido, sostiene: "La filosofía social, tal como lo vimos, comporta una disposición polémica frente al positivismo. Éste sólo ve lo particular, y en el ámbito de lo social tan sólo ve al individuo y las relaciones entre individuos, pues todo en el positivismo se agota en los hechos" (Horkheimer 2015 [1931], p. 217). En contraposición a otorgar un valor absoluto al papel del hecho en la investigación social, estima que la filosofía considera los hechos en su relación con "las ideas, esencias, totalidades independientes, esferas del Espíritu Objetivo, unidades de sentido, identidades 
nacionales, etc., que considera elementos igualmente fundamentales e incluso más auténticos del ser que los propios hechos" (Horkheimer 2015 [1931], p. 217).

Al estudio social "positivo", que ha separado claramente la metafísica de la ciencia, Horkheimer opondrá la necesidad de integrar dialécticamente la tarea de la filosofía y la de las ciencias empíricas:

Esta concepción según la cual el investigador social ve la filosofía como una actividad quizás bonita e interesante pero científicamente infructuosa por no estar sometida al control empírico, mientras que el filósofo, por su parte, se emancipa del investigador empírico porque no puede esperar por él antes de enunciar sus conclusiones totalizantes, es actualmente superada por la idea de una permanente interacción dialéctica entre la teoría filosófica y la praxis científica. (Horkheimer 2015 [1931], p. 219)

En síntesis, en Horkheimer el positivismo ${ }^{3}$ aparece asociado a:

i) el individualismo (fenómenos como unidades de análisis) de la sociedad capitalista y la actitud optimista ante el progreso que enlaza ciencia, técnica y crecimiento industrial;

ii) el naturalismo, el cual obvia el momento interpretativo en la investigación científica y olvida el papel de la subjetividad como productora de conocimiento.

2.2. Análisis de la recepción del positivismo en Horkheimer 2000 $[1937]^{4}$

Como hacen notar Leyva y Mesquita Sampaio, varios de los rasgos señalados en Horkheimer 2015 [1931] reaparecen en Horkheimer 2000 [1937]. La escisión entre "el saber formulado conceptualmente" y el "estado de cosas" se vuelve central en el análisis, sobre todo en relación con la explicación científica y el papel de la teoría en la investigación. "Explicación” y "teoría" son términos muy cercanos en lo que

${ }^{3}$ Horkheimer usa el término en este texto sin referencias a autores determinados. Podemos inferir, por el momento en que escribe, que la referencia es genérica y abarca los sentidos (a) y (b) mencionados.

${ }^{4}$ Los textos que analizaremos a continuación (Horkheimer 2000 [1937] y Horkheimer 2002 [1937]) se publicaron originalmente en el número 6 de la revista del Instituto de Investigación Social Zeitschrift für Sozialforschung. En continuidad con interpretaciones como la de O’Neill y Uebel, mostraremos cómo estas obras representan un punto de inflexión en la historia de la recepción del Círculo de Viena (O’Neill y Uebel 2004, p. 75).

Revista de Filosofía Diánoia, vol. 64, no. 83 (noviembre de 2019-abril de 2020) e-ISSN: 1870-4913 • DOI: https://doi.org/10.22201/iifs.18704913e.2019.83.1717 
se considera una perspectiva clásica, donde "explicación" se caracteriza como subsunción de un estado de cosas determinado bajo la estructura conceptual del saber teórico (Leyva y Mesquita Sampaio 2012, p. 275). Justamente Horkheimer 2000 [1937] comienza con una caracterización de la noción positivista de teoría. Según el texto, se trataría de un conjunto de proposiciones conectadas entre sí según una estructura deductiva, de modo que a partir de los enunciados que sirven de base (axiomas) se pueden seguir los restantes. Cuanto más económico y simple sea el sistema, tanto mejor será la teoría. A continuación, se enfatiza que los enunciados derivados de los axiomas deberán concordar con los hechos o acontecimientos. Si esto fracasa y se produce una contradicción entre la experiencia y la teoría, habrá que revisar una u otra. Así, Horkheimer enfatiza el control fáctico que exige la concepción positivista de la investigación científica y que es necesario para la revisión de los resultados teóricos; asimismo, subraya el carácter de provisionalidad de la teoría, que queda también manifiesto acercando "teoría" a la noción de construcción hipotética.

A los rasgos mencionados se suma el ideal de matematización o, de modo más general, de formalización:

Si se puede hablar de que esta concepción tradicional de la teoría muestra una tendencia, ésta apunta a un sistema de símbolos puramente matemático. Como elementos de la teoría, como partes de las conclusiones y proposiciones, cada vez intervienen menos nombres de objetos de experiencia, siendo sustituidos por símbolos matemáticos. Incluso las propias operaciones lógicas están ya hasta tal punto racionalizadas, que al menos en gran parte de la ciencia natural la expresión de las teorías se ha convertido en una construcción matemática. (Horkheimer 2000 [1937], p. 25)

De este modo queda caracterizada la imagen de la "teoría tradicional", la cual, según Horkheimer, sería propia de muchos modelos epistemológicos que abarcan no sólo al neopositivismo, sino también a la fenomenología husserliana y la cual se emplearía tanto para las ciencias naturales como para las del hombre y de la sociedad. El autor recalca que esta concepción de teoría no incluye el ámbito de sus aplicaciones prácticas y que también le son ajenos el origen social de los problemas y el de los fines que la sustentan. En contraposición, Horkheimer sostiene que esos esquemas conceptuales, de hecho, se producen con vistas a su aprovechamiento: "La relación de las hipótesis con los hechos no se cumple en último término en la cabeza del investigador sino en la 
industria" (Horkheimer 2000 [1937], p. 31). En relación con esto, reconoce que algunas posiciones sí han atendido a la dimensión práctica de la teoría: "Entre las diversas escuelas filosóficas, los positivistas y los pragmatistas parecen particularmente atentos al entrelazamiento del trabajo teórico con el proceso vital de la sociedad" (Horkheimer 2000 [1937], pp. 30-31).

Una crítica al modelo caracterizado como "teoría tradicional" tiene que ver con el modo en que concibe el conocimiento en general. Según Horkheimer, un rasgo característico de la teoría tradicional es su concepción de conocimiento objetivo. Según ésta, el mundo perceptible se le presenta al científico como un compendio de facticidades externas que se deben recibir y organizar sin la mediación de interpretaciones ni valoraciones, las cuales se consideran elementos subjetivos. En oposición, la "teoría crítica" se enmarca en la tradición crítica, originada por Kant, que sostiene que el conocimiento supone la acción de un sujeto. En contraposición a la "idolatría del hecho", desde la perspectiva crítica, se ve el acontecimiento, en su calidad de estado de cosas dado a la percepción, como un producto humano. En continuidad con Hegel y Marx, Horkheimer entiende que ni las formas de percepción ni lo percibido son algo natural sino histórico, constituido por la actividad del hombre. En el caso del marxismo, el mundo se ve como producto de una praxis social general (Leyva y Mesquita Sampaio 2012, p. 37).

Otro de los aspectos contra los que se dirige la crítica de Horkheimer es que la teoría tradicional "es el resultado de una abstracción que parte de la actividad científica tal como se lleva a cabo en un nivel dado de división del trabajo" (Horkheimer 2000 [1937], p. 32) en la medida en que desconoce su propia emergencia sociocultural. En consecuencia, la crítica se desliza luego al papel del científico y su desdoblamiento activo: por un lado, qua científico experto en un área del saber, considera la realidad social y sus productos "en cuanto que" algo externo e independiente y defiende sus intereses en esa realidad social a través de su participación política "en cuanto que" ciudadano. La posición crítica busca, entonces, superar esa tensión (Horkheimer 2000 [1937], p. 44).

La tarea de la teoría tradicional se enlaza con el ideal explicativo y predictivo y con el apoyo en las herramientas metodológicas, entre ellas la lógica formal, la cual se presenta como una técnica intelectual propia de la labor en marcha de la ciencia, concebida desde esta perspectiva. En contraste, la labor del teórico crítico no puede reducirse a esto, sino que su fin es "la lucha, a la que pertenece su pensamiento, y no el pensamiento como algo independiente o que se pueda separar de la lucha" (Horkheimer 2000 [1937], p. 49). La labor de la teoría con- 
lleva la dimensión práctica, inherente a sus fines: la toma de conciencia crítica con miras a la emancipación social. En este sentido, la teoría crítica se concibe como una forma de hacer ciencia que no se agota en tareas meramente teóricas, sino que se constituye en un asunto teóricopráctico. En esto, como en otros aspectos referidos, se reconoce como deudora de la idea de praxis marxista.

Con esta finalidad como telos de la labor teórica, el trabajo del científico social tradicional se ve como reproductor de la sociedad actual, ya que ni su interés explicativo y descriptivo ni su análisis acrítico de las categorías disponibles pueden favorecer el cambio social. En oposición a estas limitaciones, sostiene:

También los intereses del pensamiento crítico son universales, pero no están universalmente reconocidos. Los conceptos que surgen bajo su influjo critican el presente. Las categorías marxianas de clase, explotación, plusvalor, beneficio, de pauperización o hundimiento son momentos de una totalidad conceptual cuyo sentido no se debe buscar en la reproducción de la sociedad actual, sino en su transformación en la dirección de la justicia. (Horkheimer 2000 [1937], p. 53)

El ideal teleológico de la teoría crítica la coloca de lleno en el plano valorativo. Que el motor de la teoría sea la reversión del orden social implica la imbricación de valores en la teoría, como equidad y justicia (Horkheimer 2000 [1937], p. 77). En contraste, se endilga a la teoría tradicional la mentada "neutralidad valorativa", a la que se responde con un análisis del papel de los intereses en la investigación social: "No hay teoría de la sociedad (ni siquiera la de los sociólogos inductivistas) que no contenga intereses políticos, cuya verdad se debe determinar en la actividad histórica concreta, en lugar de hacerlo en una reflexión aparentemente neutral que, por su parte, no piensa ni actúa" (Horkheimer 2000 [1937], p. 56). La concepción de la praxis teórica según la cual la teoría tiene efectos necesarios sobre la realidad conduce a Horkheimer a pensar que hay una valoración implícita en toda teoría. En la medida en que no existe una teoría libre de valoraciones, exhorta a los científicos a ser conscientes de tal contenido, es decir, a adoptar una actitud crítica respecto de él. Tras enfatizar lo anterior, el texto se torna cuestionador de las actitudes que desconocen dicha labor crítica y colocan la tarea de la ciencia en una esfera cerrada y separada de la totalidad social, de manera que termina acusándolas de haber "renunciado ya a la humanidad" (Horkheimer 2000 [1937], p. 76).

Por lo anterior, en Horkheimer 2000 [1937] el positivismo queda englobado en la visión de la teoría tradicional según la cual:

Revista de Filosofía Diánoia, vol. 64, no. 83 (noviembre de 2019-abril de 2020) e-ISSN: 1870-4913 • DOI: https://doi.org/10.22201/iifs.18704913e.2019.83.1717 
i) la ciencia busca explicar y predecir fenómenos;

ii) los fenómenos son algo dado, externo e independiente de la acción subjetiva individual y social;

iii) en consecuencia, adopta una concepción objetivista del conocimiento científico —que, como mostraremos más adelante, es una visión muy sesgada de objetivismo-;

iv) la actividad científica se piensa como algo independiente de la totalidad social y en consecuencia se considera a sí misma autónoma, autosuficiente y neutral;

v) a la ciencia como actividad no le corresponde ninguna autocrítica, concebida como crítica de su constitución;

vi) la labor científica no se realiza en un trasfondo histórico-social.

Se sigue de lo anterior que la ciencia concebida así no logra percibir el potencial socialmente transformador que la teoría podría tener para la constitución de una sociedad más justa y equitativa.

\subsection{Análisis de la recepción del positivismo en Horkheimer 2002 [1937]}

En el tercer texto (Horkheimer 2002 [1937]) encontramos referencias a numerosos filósofos y científicos que se relacionan con el positivismo lógico, como sus antecedentes remotos o inmediatos (Leibniz, Hume, Ernst Mach, Max Planck); además, a diferencia de los dos ensayos anteriores, encontramos varias citas de autores pertenecientes al positivismo lógico o relacionados estrechamente con éstos. ${ }^{5}$ Cabe acotar que

${ }^{5}$ Las citas que hace Horkheimer son: B. Russell, "The Congress of Scientific Philosophy", cit. en Horkheimer 2002 [1937], p. 140; L. Wittgenstein, Tractatus Logico-Philosophicus, cit. en Horkheimer 2002 [1937], p. 144; H. Hahn, "Logik, Mathematik und Naturerkennen", cit. en Horkheimer 2002 [1937], p. 151; de R. Carnap, "Die alte und die neue Logik", cit. en Horkheimer 2002 [1937], pp. 169, 175 y 177; Logical Syntax of Language, cit. en Horkheimer 2002 [1937], pp. 142 y 144; The Unity of Science, cit. en Horkheimer 2002 [1937], pp. 147 y 148; Abriss der Logistik, cit. en Horkheimer 2002 [1937], p. 169, y "Logic", que apareció en Factors Determining Human Behavior, cit. en Horkheimer 2002 [1937], p. 182; de M. Schlick, "Philosophie und Naturwissenschaft", cit. en Horkheimer 2002 [1937], p. 179; de H. Weyl, Philosophie der Mathematik und Naturwissenschaft, cit. en Horkheimer 2002 [1937], pp. 144 y 190 (que es el único texto de un positivista lógico y lo cita también en Horkheimer 2000 [1937]). Las citas de Neurath pro- 
el artículo de Otto Neurath "Inventory on the Standard of Living" apareció en el mismo número de Zeitschrift für Sozialforschung que los dos ensayos de Horkheimer del mismo año. ${ }^{6}$

Coincidimos con O'Neill y Uebel en que no se encuentran diferencias importantes entre los positivismos de Comte, Mach y el Círculo de Viena en Horkheimer 2002 [1937]. Por otra parte, los autores señalan que la idea de homogeneidad dentro del Círculo de Viena es errónea (O’Neill y Uebel 2004, pp. 78 y 80). Lo mismo sostienen Araujo y Medina, quienes agregan, respecto de la polémica entre Horkheimer y Neurath, que este último es el miembro del Círculo de Viena que menos representa las posiciones criticadas (Araujo y Medina 2014, p. 117).

\subsubsection{Caracterización general del positivismo lógico. Rasgos centrales}

Comencemos por el análisis de los rasgos caracterizadores del positivismo lógico que hallamos en el texto. Primero consideraremos la concepción de la teoría tradicional y su papel en la investigación científica. Horkheimer afirma:

El positivismo de hoy en día suele ubicar sus orígenes en Hume, por un lado, y en Leibniz, por otro. Combina el empirismo escéptico con una lógica racionalizada, a la que espera volver más fructífera para la ciencia. El ideal que persigue es el conocimiento en la forma de una ciencia universal formulada matemáticamente deducible desde el número más reducido posible de axiomas, un sistema que asegura el cálculo de la aparición de todos los sucesos. (Horkheimer 2002 [1937], p. 138) ${ }^{7}$

vienen de "Soziologie im Physikalismus", cit. en Horkheimer 2002 [1937], p. 147; "L'Encyclopédie comme 'modèle'", cit. en Horkheimer 2002 [1937], pp. 146, 163 y 165; Empirische Soziologie, cit. en Horkheimer 2002 [1937], pp. 152-153; además, el autor remite a H. Reichenbach, "Die philosophische Bedeutung der modernen Physik", cit. en Horkheimer 2002 [1937], p. 182.

${ }^{6}$ Neurath se incomodó mucho por la opinión que allí se manifestaba sobre el positivismo lógico y le respondió al editor de la revista de inmediato. Luego le hizo llegar a Horkheimer un nuevo ensayo con el título de "Entwurf", donde defendía el positivismo lógico de las acusaciones de Horkheimer. En las cartas que intercambiaron entre 1937 y 1938, tras la publicación de Horkheimer 2002 [1937], Neurath insistió en el derecho a la publicación de su respuesta en el siguiente número de la revista, pero Horkheimer desestimó ese pedido y le recomendó que intentara publicarla en una revista más afín a la temática que allí aborda, como Erkenntnis (Horkheimer 1985-1996, vol. 16).

${ }^{7}$ Las citas de Horkheimer 2002 [1937] son traducciones nuestras a partir de la versión registrada en la bibliografía.

Revista de Filosofía Diánoia, vol. 64, no. 83 (noviembre de 2019-abril de 2020) e-ISSN: 1870-4913 • DOI: https://doi.org/10.22201/iifs.18704913e.2019.83.1717 
A continuación agrega: "La sociedad, también, se tiene que explicar de esta manera"; de este modo, enfatiza la idea de unidad metodológica que caracteriza a la posición (Horkheimer 2002 [1937], p. 138).

Sobre la base de esta organización teórica, moldeada a partir de la idea de cálculo lógico deductivo, el fin de la teoría está en la explicación y predicción de los sucesos, con cierto grado de probabilidad en su aparición. Aquí también se enfatiza que este ideal vale tanto en el ámbito de las ciencias naturales como en el de las sociales (Horkheimer 2002 [1937], p. 139).

Otro rasgo que reaparece en la caracterización del movimiento en cuestión es - como en los textos de Horkheimer abordados en las secciones anteriores - la creencia en la observación empírica como única vía para la justificación de nuestro conocimiento científico. Sobre esta base se defiende la objetividad científica y se oculta el papel constitutivo de los factores subjetivos, históricos y sociales en la composición del conocimiento científico (Horkheimer 2002 [1937], pp. 141, 178 y 200).

El empirismo lógico tiene esto en común con el empirismo anterior: ambos sostienen que todo conocimiento respecto de los objetos se deriva, en definitiva, de los datos de la experiencia sensorial. Así, Carnap piensa que todos los conceptos "son reductibles a conceptos más básicos relacionados con los datos dados, el contenido inmediato de la experiencia". (Horkheimer 2002 [1937], p. 141) ${ }^{8}$

Según el autor, "la verificación mediante la percepción es el alfa y omega del empirismo. Se ata a lo que es, a la garantía de los hechos." Y para dar testimonio de esta afirmación recurre a un texto de Wittgenstein: "El mundo es todo lo que es el caso [...] El mundo se divide en hechos". Ésta —según la interpretación decididamente cuestionable de Horkheimer - es la visión que se expresa en el mayor trabajo del empirismo moderno (Horkheimer 2002 [1937], p. 141). ${ }^{10}$

Además, le atribuye al positivismo lógico una concepción inductivista: "Respecto del futuro, la actividad característica de la ciencia no

${ }^{8}$ R. Carnap, "Die alte und die neue Logik", Erkenntnis, vol. 1, 1930-1931, p. 24, cit. en Horkheimer 2002 [1937], p. 141.

${ }^{9}$ L. Wittgenstein, Tractatus Logico-Philosophicus, Kegan Paul, Londres, 1922, p. 21, cit. en Horkheimer 2002 [1937], p. 144.

${ }^{10}$ En esta apreciación respecto de la obra de Wittgenstein se denota que Horkheimer no logra captar la idea wittgensteiniana de "hechos en el espacio lógico", de otro modo, no se comprendería la asignación de "empirista" a Wittgenstein. Esta dificultad también puede trasladarse a la interpretación de la posición de Carnap.

Revista de Filosofía Diánoia, vol. 64, no. 83 (noviembre de 2019-abril de 2020) e-ISSN: 1870-4913 • DOI: https://doi.org/10.22201/iifs.18704913e.2019.83.1717 
es la construcción, sino la inducción. Mientras más veces haya ocurrido algo en el pasado, lo más probable será que ocurra en el futuro. El conocimiento se relaciona sólo con lo que es y con su recurrencia" (Horkheimer 2002 [1937], p. 144).

Esa atribución de inductivismo no resulta coherente con muchas otras caracterizaciones epistemológicas en las que el autor le adjudica una organización hipotética a la actividad científica desde la mirada del neopositivismo:

El empirismo [...] incansablemente declara su intención de hacer a un lado cualquier convicción si nueva evidencia la falsea. "Ninguna regla del lenguaje físico es definitiva" y "la prueba se dirige a la base, no a una hipótesis aislada, sino a todo el sistema de la física como un sistema de hipótesis" (Horkheimer 2002 [1937], p. 144). ${ }^{11}$

Sin embargo: "lo que vale para tomar esa decisión es la conveniencia, que escapa a toda determinación teórica. ${ }^{12}$ El empirismo niega que el pensamiento pueda evaluar las observaciones y la manera en que la ciencia las combina" (Horkheimer 2002 [1937], p. 144), de manera que opone los criterios de confirmación empírica - propios de los neopositivistas - a aquellos que superan la dependencia de los resultados fácticos como único criterio de justificación. Ese carácter provisional de las teorías científicas y el alegado objetivismo redundarán, según Horkheimer, en las consecuencias práctico-políticas que abordaremos en la sección 3.ii.

El empirismo, es verdad, incansablemente declara su intención de hacer a un lado cualquier convicción si una nueva evidencia la falsea. [...] Sin embargo, el empirismo limita estas pruebas a puntos de vista neutrales, objetivos, no normativos, es decir, a perspectivas que están, en definitiva, aisladas. (Horkheimer 2002 [1937], p. 144)

${ }^{11}$ R. Carnap, Logical Syntax of Language, trad. A. Smeaton, Kegan Paul, Londres, 1937, p. 318, cit. en Horkheimer 2002 [1937], p. 144.

${ }^{12}$ Aquí Horkheimer explica en una nota: "El empirismo lógico coincide con la teoría dominante en la actualidad en que sostiene que la resolución misma del conflicto entre el hecho y la teoría no se puede formular teóricamente. 'Aquí es donde el genio deviene su propio dueño' es la explicación que ofrece H. Weyl, Philosophie der Mathematik und Naturwissenschaft, en Handbuch der Philosophie II, Múnich/Berlín, 1927, p. 113" (Horkheimer 2002 [1937], p. 144).

Revista de Filosofía Diánoia, vol. 64, no. 83 (noviembre de 2019-abril de 2020) e-ISSN: 1870-4913 • DOI: https://doi.org/10.22201/iifs.18704913e.2019.83.1717 
De este modo, la tarea legítima de la investigación científica se centra en la predicción de sucesos de acuerdo con leyes determinadas. La ciencia empirista reduce la situación de que $B$ sea una causa de $A$ al hecho de que $B$ sea el resultado buscado de una acción intencional de $A$ y, así, se pierde de vista la estructura específica de la relación entre sujeto y objeto: "Así como es posible predecir las acciones de los individuos mediante métodos idénticos a los de la predicción en los procesos físicos, también es posible hacer predicciones sobre grupos sociales. La teoría empirista es 'behaviorismo social'" (Horkheimer 2002 [1937], p. 152).

Según Horkheimer, el empirismo rechaza en general la noción de sujeto. Así, por ejemplo, el behaviorismo desarrolla una psicología a partir de los conceptos y métodos de las ciencias que se ocupan de la materia inorgánica. De este modo, se anula la dimensión intencional de la conducta humana y no se vislumbra la diferencia cualitativa entre causa y motivo. En este sentido hay que entender la siguiente cita de Russell: "Cuando un adulto piensa en un acto, pero no lo ejecuta, su falla depende únicamente del hecho de que otros pensamientos o circunstancias están presentes e interfieren con el actuar" (Horkheimer 2002 [1937], pp. 149-150).

La siguiente crítica que consideraremos se dirige al carácter proposicional de la actividad científica, y Horkheimner la introduce en la argumentación a través de una comparación entre el empirismo moderno y el contemporáneo. Según él, en el empirismo moderno: "Las ciencias aparecían como una forma condensada del conocimiento proveniente de las experiencias diarias del individuo", con lo cual, "su filosofía contiene este elemento dinámico: la relación con un sujeto cognoscente" (Horkheimer 2002 [1937], p. 142). Puesto que el positivismo del siglo xx busca eliminar todo lo que haya de subjetivo en la teoría, esa unidad entre el conocimiento y la experiencia subjetiva se pierde. "La ciencia y, por lo tanto, la filosofía científica tratan sobre el mundo dado sólo en la forma de proposiciones acerca de ese mundo" (Horkheimer 2002 [1937], p. 143). También sostiene que, a pesar del afán por deshacerse de todo rastro de subjetividad en la teoría, las tendencias sociales e individuales no pueden dejar de filtrarse al aparato conceptual del empirista (Horkheimer 2002 [1937], p. 153).

A su vez, el contenido proposicional está interpretado físicamente, lo cual - como mostraremos- constituye una versión interpretativa del fisicalismo. De este modo, Horkheimer cita a Carnap:

La longitud de una calle, la temperatura de un cuerpo y la frecuencia de una oscilación nunca se conciben en física como cuestiones subjetivas ni, 
por lo tanto, como desacuerdos irresolubles [...]. Los físicos creen que, cuando tal acuerdo no se alcanza en la práctica, se debe a dificultades técnicas (imperfección de los instrumentos, falta de tiempo, etc.) [...] Las determinaciones físicas son válidas intersubjetivamente. ${ }^{13}$

Esta interpretación "fisicista" de la experiencia se traslada al tratamiento de los enunciados protocolares en el ámbito de las ciencias sociales, ya que no se trabaja directamente con percepciones, sino más bien con hechos formulados en juicios (Horkheimer 2002 [1937], pp. 143-144).

Así, cuando Horkheimer aborda las proposiciones protocolares enfatiza que la teoría científica opera con algo que es resultado de una mediación. Lo que reclama Horkheimer es que hay que darse cuenta de qué significa esa mediación y que se recupere una conexión más inmediata con lo que aún se considera no racionalizado científicamente. Parece sostener que la única forma de tener experiencia del mundo no es la mediación del lenguaje y, por lo tanto, que hay otras formas de producir conocimiento.

Sin embargo, la interpretación de Horkheimer erra al no distinguir entre la propuesta de Carnap y la de Neurath, sino que las unifica en una sola perspectiva (asimilando la última a la primera). Como hace notar Prono:

Desde esta perspectiva, la contribución de Neurath puede ser vista como una "pragmática de la ciencia" (en términos de Neurath "behavouristics of scholars"), en oposición o en paralelo a la "lógica de la ciencia" carnapiana. Mientras esta última brinda herramientas sintácticas y semánticas para analizar la estructura de las teorías científicas y la relevancia de la evidencia empírica, valiéndose de los recursos de la lógica de la investigación científica, la primera se interesa por integrar en su análisis aportes empíricos provenientes de la psicología, la sociología y la historia de la ciencia. La tarea de la pragmática de la ciencia no se reduce a su aspecto descriptivo, sino que conserva una dimensión normativa en la medida en que exige la explicitación de los procesos de aceptación y rechazo de pretensiones cognoscitivas. (Prono 2010b, p. 1)

Resumamos los rasgos centrales de la caracterización del positivismo en el texto analizado:

i) el positivismo ve en la ciencia, en particular en la metodología científica, sobre todo una actividad cognitiva cuyos objetivos principales son la explicación y la predicción de los fenómenos;

${ }^{13}$ R. Carnap, The Unity of Science, trad. M. Black, Kegan Paul, Londres, 1934, p. 64, cit. en Horkheimer 2002 [1937], p. 148.

Revista de Filosofía Diánoia, vol. 64, no. 83 (noviembre de 2019-abril de 2020) e-ISSN: 1870-4913 • DOI: https://doi.org/10.22201/iifs.18704913e.2019.83.1717 
ii) en esta empresa la "fe" en el conocimiento de los hechos en sí es prioritaria (empirismo radical) lo que se liga, a su vez, con la confianza en la posibilidad de acceder proposicionalmente al conocimiento del mundo fáctico a través de su captación lógicomatemática;

iii) en esta tendencia, el positivismo aspira a la objetivación del mundo fenoménico, para lo cual debe aislar los elementos subjetivos constituyentes (tanto en el nivel individual como en el social);

iv) así como el físico observa los hechos del mundo físico, los científicos sociales observan los hechos sociales qua hechos individuales sujetos a regularidades legales. En el estudio de las sociedades capitalistas estos hechos son los individuos aislados, a los que se accede naturalizándolos y volviéndolos "cosas en sí", fuera de las dimensiones sociales e históricas que los constituyen;

v) el positivismo ha disociado la tarea filosófica de la tarea científica y ha restringido la labor de la primera a una revisión metateórica de la segunda, con lo que ha abandonado la tarea autorreflexiva propia de la filosofía;

vi) por último, esta posibilidad interna de la concepción positivista no es lo único que la vuelve ineficaz para el cambio social, sino también sus ideales de progreso social, expresados en la aplicación de la ciencia al mejoramiento de la técnica y la industria. Esos ideales, sumados al carácter acrítico de su procedimiento, impiden al positivismo percibir la verdad del mundo (la situación de opresión y explotación de la sociedad capitalista).

2.3.2 Más sobre las estrategias de Horkheimer para la construcción antagonista del positivismo

Analicemos con mayor detalle la estrategia del autor para lograr la caracterización que le permite señalar los límites y las dificultades del positivismo en general y de sus enfoques sociales en particular. Esta estrategia no se enmarca en un ideal de caracterización epistemológica per se, sino en un eje de valoración práctico-política sobre el positivismo.

Así, podemos reconstruir la vía estratégica de Horkheimer: (i) se resalta la "idolatría del hecho", que se asigna como rasgo central del positivismo. Esto conduce directamente a un objetivismo, a partir del cual (ii) se eliminan los elementos subjetivos del conocimiento; la consecuencia de esto es: (iii) la producción de un conocimiento científico 
con resultados útiles pero con una visión sesgada de la realidad a la que se dirigen. El positivismo, alentado por la utilidad de los resultados a los que llega la ciencia, es optimista en relación con el progreso científico y tecnológico, y busca expandir su método hasta alcanzar la unidad de las ciencias. A partir de estas observaciones, Horkheimer extrae su valoración ético-política sobre los supuestos y las consecuencias del positivismo: (iv) el ocultamiento de la verdad por la "idolatría del hecho" no permite conocer el contexto de explotación y dominación que subyace en el orden social, y (v) el optimismo en el papel de la ciencia y de la técnica en la sociedad capitalista contribuye a perpetuar el orden vigente.

Como comentamos, a (i) le sigue la asignación de objetivismo (que abordaremos más adelante, en la sección 3.i), que se caracteriza por (ii) un antisubjetivismo en relación con las condiciones de producción del conocimiento en lo que atañe al sujeto individual y al sujeto social:

El empirismo sostiene que tales ideas surgen al confundir los deseos personales, las creencias morales y los sentimientos con la ciencia; considera que la separación entre los valores y la ciencia es uno de los logros más importantes de la ciencia moderna. [...] La concepción de una teoría que se rige enteramente por un interés, según este argumento, es incompatible con una ciencia objetiva. (Horkheimer 2002 [1937], p. 164)

En el mismo orden temático hallamos el siguiente texto:

En los ojos del empirista, la ciencia no es más que un sistema de acomodamientos y desacomodamientos de hechos y no importa qué hechos se seleccionan del número infinito presente de ellos. [...] Este proceso, que antes se identificaba con la actividad del entendimiento, está desconectado de cualquier actividad que pudiera afectarlo y, por lo tanto, que modificara su dirección y significado. Todo lo que el idealismo designa como idea y como fin y que el materialismo considera práctica social y actividad histórica consciente [...] el empirismo no lo admite en absoluto como condición del conocimiento (Otto Neurath). ${ }^{14}$ No hay una forma de pensamiento que esté adaptada a los métodos y resultados de la ciencia y entrelazada con intereses definidos que pueda criticar las formas conceptuales y el patrón estructural de la ciencia, a pesar de que la ciencia depende de todas estas cosas. (Horkheimer 2002 [1937], pp. 145-146)

${ }^{14}$ Horkheimer atribuye estas ideas a Otto Neurath y en una nota a pie especifica: cfr. O. Neurath, "Soziologie im Physikalismus", Erkenntnis, vol. 12, 1931, pp. 393431, en esp. pp. 423-428, y O. Neurath, Empirische Soziologie, Springer, Viena, 1931, pp. 128-147, cit. en Horkheimer 2002 [1937], p. 145.

Revista de Filosofía Diánoia, vol. 64, no. 83 (noviembre de 2019-abril de 2020) e-ISSN: 1870-4913 • DOI: https://doi.org/10.22201/iifs.18704913e.2019.83.1717 
Luego de esta valoración, aparecerá la de la reducción de los sujetos a hechos y de las acciones humanas (en especial, las intencionales-volitivas) a comportamientos, regularidades causales observables (como apuntamos en la sección 2.3.1, la ciencia social se convierte en un "behaviorismo social", según Horkheimer). De acuerdo con esta lectura, esa tendencia busca desarrollar una psicología a partir de los conceptos y métodos de las ciencias que se enmarcan en un modelo de explicación reduccionista. Se anula la dimensión intencional de la conducta humana y no se vislumbra la diferencia cualitativa entre causa y motivo.

Horkheimer resalta el carácter instrumental del positivismo en el sentido (iii): el ideal explicativo-predictivo, que concibe el objeto cognitivo (los sujetos sociales) como mero factum cuyas acciones pueden colocarse en patrones repetitivos, empíricamente controlables. En muchos pasajes se observa que la absolutización del hecho y su autonomía de cualquier determinación conceptual se asocian con la concepción de una ciencia social instrumental explicativa y predictiva:

No hay más que hechos, y la totalidad del aparato conceptual de la ciencia sirve para determinarlos y predecirlos. Cuando la relación de la conciencia con el mundo objetivo entra en consideración, suele abordarse como una colección de hechos, como hábitos condicionados psicológicamente o de alguna forma similar. Cualquier otra forma de consideración carece de sentido. (Horkheimer 2002 [1937], p. 154)

En la apreciación de Horkheimer, los hechos, convertidos en "hechos en sí", y los objetivos explicativos y predictivos de los hechos en sí imponen un límite a las tendencias positivistas: no pueden ir más allá de ellos. En seguida, se considera que el positivismo no puede llevar a cabo una autocrítica de la práctica científica en esta línea de naturalización del conocimiento fáctico. Según el autor, este último queda reducido a sus aspectos físicos y fisiológicos y separado de su proceso de constitución sociohistórica y de la relación con los componentes conceptuales que interactúan con él (Horkheimer 2002 [1937], p. 146). En consonancia con lo que sostenía en la conferencia de 1931, esta dificultad no existe en una investigación de base hegeliana:

Podemos separar la ciencia de otras esferas de la vida social; se puede concebir que la ciencia consiste en la determinación y predicción de hechos. Sin embargo, debería saberse, por lo menos desde la Fenomenología del espíritu de Hegel, que las experiencias, sensaciones y percepciones tal como 
nos son dadas resultan ser extremas sólo para una comprensión limitada, y realmente son derivadas y dependientes. (Horkheimer 2002 [1937], p. 157)

La concepción positivista, tras devenir un "totalitarismo del hecho científico" y al no poder ir más allá de los datos puros, no logra alcanzar un nivel reflexivo que le permita captar la actividad subjetiva en la constitución y el conocimiento de aquéllos.

En consecuencia, Horkheimer considera que el positivismo falla en su modo de acceder al conocimiento de la realidad social. Aunque acepta que el empirismo demostró su capacidad para producir un conocimiento que nos es útil en la actualidad, sostiene que la metafísica penetra más profundo que él en la realidad. "Sin embargo, el positivismo lógico estigmatiza como metafísica a todo pensamiento que intente clarificar esas relaciones y a todas las teorías que puedan tomar una posición crítica respecto de las ciencias especiales" (Horkheimer 2002 [1937], p. 185). Lo que motiva la preocupación de Horkheimer es que los resultados (útiles) de la ciencia positivista se alcanzan sólo a costa de renunciar al conocimiento de la verdad, sobre todo, en relación con las condiciones sociales de dominación propias del sistema capitalista. La investigación social positiva no logra explicar el sometimiento que sufren los hombres ni los mecanismos de opresión que operan en la sociedad. Por ende, tampoco es capaz de revertirlos. He aquí la presencia del juicio práctico-valorativo (iv).

Se resalta en la interpretación de Horkheimer que la concepción de los hechos y de la investigación fáctica para el positivismo acarrea la idea de que los hechos considerados en sí mismos son "apariencias de la sociedad que disfrazan las cosas" (O’Neill y Uebel 2004, p. 81), de manera que la fetichización del hecho se asocia con la idea de ocultamiento de la verdad y, en consecuencia, con la imposibilidad de toda crítica y acción trasformadora.

A continuación, se estima que lo que motiva al positivismo a persistir en la defensa y el empleo de estos métodos radica en la relación con el progreso técnico y tecnológico que ofrecen. Sin embargo, Horkheimer advierte que se trata de un optimismo ingenuo que sólo conduce a la perpetuación del orden vigente. (v) En este caso, también sobreviene la valoración práctico-política: el positivismo "le asigna una autoridad intelectual suprema a la ciencia acreditada, cuya estructura dada y métodos están conciliados con las condiciones existentes" (Horkheimer 2002 [1937], p. 144). Según Horkheimer (Horkheimer 2002 [1937], p. 164) este optimismo impide al empirismo lógico brindar las herra-

Revista de Filosofía Diánoia, vol. 64, no. 83 (noviembre de 2019-abril de 2020) e-ISSN: 1870-4913 • DOI: https://doi.org/10.22201/iifs.18704913e.2019.83.1717 
mientas necesarias para enfrentar el viraje del capitalismo al fascismo (Horkheimer 2002 [1937], p. 161), pues su confianza en la educación y la ciencia como medios para lograr un mundo social mejor, así como su fe ilustrada en el progreso, lo tornaron incapaz de ver las consecuencias peligrosas del desarrollo del sistema capitalista. Para Horkheimer, lo que guía a la ciencia son la lógica burguesa de dominio y la racionalidad instrumental.

El optimismo que hemos comentado conduce a perpetuar el uso de los mismos métodos, pero también a intentar expandirlos hacia todos los ámbitos. En ese sentido, Horkheimer cuestiona el ideal del proyecto de la ciencia unificada, pues estima que con él se pretendía alcanzar la unidad mediante un lenguaje depurado de toda carga subjetiva e ideológica que garantizara la objetividad empírica del científico. De nuevo, pasamos de la evaluación epistemológica a la valoración éticopolítica:

La creencia armoniosa e ingenua que subyace en su concepción ideal de la unidad de la ciencia y, en el último análisis, en todo sistema del empirismo moderno corresponde al mundo del liberalismo. [...] De acuerdo con los empiristas ésta es una "coincidencia afortunada" que no es necesario analizar para determinar su importancia y alcance. (Horkheimer 2002 [1937], p. 147)

En esta interpretación, el empirismo lógico construye una posición sobre la ciencia y su papel social que contribuye a la aceptación del status quo vigente y, así, favorece los rasgos de injusticia social del sistema predominante:

Si la ciencia en su conjunto se guía por el empirismo y el intelecto renuncia a su insistente y confiado mecanismo de investigar la maraña de observaciones para descubrir más sobre el mundo aun que la prensa diaria bien intencionada, estará participando pasivamente en el mantenimiento de la injusticia universal. (Horkheimer 2002 [1937], p. 151)

La valoración práctico-política que el autor hace del positivismo, siguiendo el orden argumentativo presentado, es que éste sólo puede "actuar en consonancia" con el sistema y el poder político dominantes y de ningún modo puede convertirse en motor de la transformación social que busque revertir las situaciones de opresión e injusticia social. 


\section{Discusión}

Una crítica central al positivismo desde la perspectiva de Horkheimer es que rara vez queda claro qué extensión abarca el término "positivismo". La única excepción es el texto Horkheimer 2002 [1937], donde las referencias al positivismo lógico son explícitas, aunque los límites de la extensión de la denominación no dejan de ser borrosos. A esa vaguedad en la extensión del término se le añade que la expresión, más que una referencia histórica a los movimientos de los siglos XIX y XX, es una construcción interpretativa de ellos.

Además, es importante señalar que en la caracterización del positivismo lógico el autor homogeneiza las diversas perspectivas de los autores representativos del movimiento o relacionados con él (Horkheimer 2002 [1937]). Recordemos que coloca a Wittgenstein y Russell en la misma perspectiva que Carnap, Hahn, Schlick y Neurath, a pesar de las diferencias que han sido reconocidas en muchos estudios.

Asimismo, hay que destacar que en la interpretación de Horkheimer es central la estrategia discursiva que lo lleva a señalar los errores y límites del positivismo y le permite exponer su propia perspectiva y mostrar que es más adecuada.

En lo que sigue, repasaremos las tesis antagónicas que podemos extraer de la interpretación de Horkheimer y haremos una crítica de ellas.

i) El positivismo presenta el conocimiento científico como conocimiento de hechos dados, independientes de la labor subjetiva; en contraposición, la visión de la Escuela de Fráncfort concibe el conocimiento de hechos desde la perspectiva de que los sujetos, como los hechos, son el resultado histórico de la acción humana.

La primera parte de esta tesis (i.a) se centra en la idea de conocimiento científico como conocimiento de hechos en sí. La segunda parte (i.b) se focaliza en la perspectiva objetivista asignada al positivismo - ya comentada varias veces- - Sobre (i.a) debemos señalar que ni siquiera el positivismo comtiano se ajusta a dicha idea. Podemos leer en el Curso de filosofía positiva:

La más importante de estas consideraciones, extraída de la naturaleza misma del tema, consiste en la necesidad experimentada en todas las épocas, de una teoría cualquiera que coordine los hechos, dada la evidente imposibilidad del espíritu humano de sistematizar una teoría, partiendo de la simple observación. (Comte 1980 [1830], p. 28) 
La función de la teoría en el conocimiento empírico, la vinculación entre la estructura lógica de ésta y los datos fácticos, así como la reflexión sobre la base conceptual y semántica de los enunciados que conforman el cuerpo teórico fueron algunos de los tópicos más trabajados desde la perspectiva del positivismo lógico, de modo que simplificar la apreciación del conocimiento según el positivismo y reducirlo al conocimiento de datos o hechos en sí no se condice de ningún modo con las obras de los miembros del movimiento.

En el caso particular de Neurath la afirmación es a todas luces una apreciación incorrecta, porque los hechos protocolares implican la acción tanto personal como social e histórica de los investigadores. Si bien Horkheimer acepta el carácter intersubjetivo de la propuesta de Neurath, no llega a discernir otros elementos que ésta involucra. Este último propone su primera versión sobre los enunciados protocolares (Neurath 1987 [1932]) y luego la retoma en otros textos, para ser reconstruida minuciosamente por Thomas Uebel (Uebel 1992, Uebel 1993 y Uebel 2009). De acuerdo con Prono 2010b, en esa versión se establece un conjunto de condiciones propias de los enunciados protocolares: se requiere que el sujeto que los expresa se halle en un estado mental como consecuencia de su interacción con un acontecimiento extralingüístico, es decir, un estado de estimulación (condición sensorial); no se requiere que tengamos evidencia directa del estado de cosas designado por la oración objeto, sino que no tengamos evidencia en contra de lo que afirma (condición de coherencia negativa); además, es necesario que la conceptualización que se utilice en la descripción del estado de cosas revele que el observador ha aprendido los conceptos relevantes de su comunidad lingüística específica y que los empleó de manera correcta y consciente en el enunciado en cuestión (condición de intencionalidad); por último, se exige la aparición pública del enunciado en cuestión para que se admita como válido y que se incorpore en el cuerpo de enunciados científicos (condición de institucionalización).

La última de las condiciones supone la aceptación general del cuerpo de conocimientos por una comunidad. Esto implica que la condición de aceptación supera las instancias subjetivas y aspira a una universalidad, la cual, aunque esté mediada por aspectos psicosociológicos, no implica ningún relativismo ni un mero consensualismo.

En relación con Carnap, desde sus primeras obras (1928), manifestó una clara preocupación por dilucidar qué se entiende por "hechos empíricos" y elaboró una teoría fenomenista; sin embargo, tiempo después abandonaría esa versión de 1928. Es destacable que en las obras de Carnap que Horkheimer 2002 [1937] enuncia cuando el autor aborda

Revista de Filosofía Diánoia, vol. 64, no. 83 (noviembre de 2019-abril de 2020) e-ISSN: 1870-4913 • DOI: https://doi.org/10.22201/iifs.18704913e.2019.83.1717 
la sintaxis lógica del lenguaje y su vinculación con el contenido empírico proposicional, no es posible hablar desde una perspectiva lógicosemántica de hechos sin predicados ni de relaciones entre hechos. La búsqueda de una isomorfía entre el mundo lógico y el empírico impone la idea de concebir los hechos ya en esta trama predicativo-relacional (cfr. Carnap 2000 [1937]).

En relación con (i.b) frente a la idea de que la ciencia desde la perspectiva positivista es el reflejo de los hechos del mundo sin participación de conceptos o categorías, debemos señalar que aun el empirismo baconiano - del cual el positivismo es heredero tanto motu proprio como según la visión de la Escuela de Fráncfort- no desconoce el papel de los conceptos en el conocimiento (ídolos del foro). De hecho, la clarificación y definición de los conceptos fue una demanda de aquel movimiento, como también lo fue del positivismo comtiano. La exigencia se corona en el positivismo lógico como la búsqueda de un sistema total de conceptos.

También es importante señalar que en Hahn, Neurath y Carnap 2002 [1929] —un texto que curiosamente Horkheimer no cita- se enfatiza la labor grupal humana, constitutiva de la ciencia:

La concepción científica [...] se propone como objetivo la ciencia unificada. El esfuerzo es aunar y armonizar los logros de los investigadores individuales en los distintos ámbitos de la ciencia. De esa aspiración se sigue el énfasis en el trabajo colectivo; de allí también la acentuación de lo aprehensible intersubjetivamente. (Hahn, Neurath y Carnap 2002 [1929], p. 112)

ii) A la perspectiva empirista y objetivista del positivismo Horkheimer le agrega la perspectiva lógica y enunciativa que caracteriza al positivismo lógico, en oposición a la perspectiva dialéctica que es propia de la concepción de la Escuela de Fráncfort.

Como vimos, la perspectiva lógico-enunciativa en el positivismo lógico se asocia al ideal de un lenguaje único, que pueda salvaguardarse de las interpretaciones múltiples y trace una línea demarcatoria entre los enunciados científicos y los que no lo son (ii.a); asimismo, la perspectiva enunciativa se relaciona con las condiciones de contrastación empírica de enunciados en la ciencia (ii.b), la cual implica, a su vez, una caracterización de aquello con lo que se contrasta un enunciado empírico, lo empírico en sí (ii.c).

La lectura oficial del ideal referido fue la versión popularizada de Ayer 1958 [1935], que Horkheimer 2002 [1937] parece compartir.

Revista de Filosofía Diánoia, vol. 64, no. 83 (noviembre de 2019-abril de 2020) e-ISSN: 1870-4913 • DOI: https://doi.org/10.22201/iifs.18704913e.2019.83.1717 
Como es conocido, uno de los problemas iniciales del positivismo lógico consiste en las posibilidades de distinguir entre los enunciados de los que puede predicarse verdad o falsedad (enunciados tautológicos y enunciados verificables empíricamente) y los enunciados "metafísicos" o pseudoproposiciones, cuya atribución de verdad o falsedad resulta imposible y, por ende, carecen de sentido. Hegselmann 1987 denomina al tópico anterior "sense theorem". Por otro lado, la negación del conocimiento a priori y la exigencia de verificación de los enunciados sintéticos como criterio de justificación se resumen, según el autor, en el "base theorem".

Así, el ideal del positivismo lógico temprano puede entenderse como el esfuerzo por que el conocimiento se circunscriba a la totalidad de las proposiciones que resultan legítimas de acuerdo con "the sense and the base theorems".

Como se mencionó antes, una de las consecuencias de lo anterior es que el lenguaje de la ciencia debe ser uno, que pueda ligarse directamente con el contenido de la experiencia fáctica (el lenguaje observacional) o que de algún modo pueda reducirse a éste. De acuerdo con Hahn, Neurath y Carnap:

La concepción científica [... ] se propone como objetivo la ciencia unificada [...] de allí surge la búsqueda de un sistema de fórmulas neutral, de un simbolismo liberado de la escoria de los lenguajes históricamente dados; y de allí también, la búsqueda de un sistema total de conceptos. (Hahn, Neurath y Carnap 2002 [1929], p. 112)

Sin embargo, la uniformidad en la resolución de qué es el lenguaje observacional y cuál es su papel en la actividad científica no es tal como Ayer y Horkheimer la juzgan, sino que tiene diversas variantes: la más importante es la de Otto Neurath, para quien el ideal no implica reconstruir el conocimiento científico en un macrosistema de enunciados con sentido. Sobre todo a partir de los años treinta, desarrollará decididamente la idea de la "ciencia unificada" en el marco de un pluralismo epistemológico. Para ello, rechaza la unidad como un sistema de enunciados científicos cuya legitimidad se alcanzaría a través del criterio verificacionista. La propuesta de Neurath virará hacia la defensa de un lenguaje fisicalista. El concepto de "ciencia unificada" requiere, en primer lugar, un compromiso con esa concepción del lenguaje; dicho compromiso constituirá la base a partir de la cual los científicos generen hipótesis diversas - plurales- que sometan al escrutinio de sus colegas

Revista de Filosofía Diánoia, vol. 64, no. 83 (noviembre de 2019-abril de 2020) e-ISSN: 1870-4913 • DOI: https://doi.org/10.22201/iifs.18704913e.2019.83.1717 
en un proceso de colaboración y negociación entre los miembros de la comunidad científica. Como hace notar Prono:

Tal lenguaje debe ser fisicalista en el sentido de que debe contener referencias al orden espacio-temporal que nos es accesible intersubjetivamente. Este rasgo proporciona un criterio de identificación de los enunciados de experiencia o enunciados protocolares. De modo que el lenguaje fisicalista no debe identificarse con el lenguaje de la física, sino como una alternativa al lenguaje fenomenalista que toma como punto de partida a las vivencias individuales de un sujeto. (Prono 2010a, p. 88)

iii) El positivismo lógico persigue el ideal de unificación de las ciencias y de homogeneidad doctrinal para sustituir la filosofía —en particular la metafísica- por la ciencia (como un todo). En contraposición, la perspectiva de la Escuela de Fráncfort apuesta por la interrelación activa entre ciencia empírica y filosofía, y recupera el papel de la metafísica en el conocimiento.

Se acepta sin discusión la asignación de "antimetafísica" a la perspectiva positivista; sin embargo, no se explicita, por lo general, en qué consiste esa posición según las diversas aristas del positivismo decimonónico y del contemporáneo. En el caso del positivismo lógico esa perspectiva se relaciona no sólo con los criterios de significación y de justificación de las proposiciones científicas antes señalados, sino también con los papeles político y social que se les asigna a las posiciones metafísicas (tal como sucede en el positivismo decimonónico). En palabras de Neurath:

Los términos metafísicos dividen, los términos científicos unen. Los científicos, unidos por un lenguaje unificado, forman un tipo de trabajadores de la república de las letras. [...] En contraposición, los filósofos son comparables con los señores feudales de San Gimignano. Se sientan en sus torres solitarias en la oscuridad de la noche y montan guardia en contra de sus vecinos, erigiendo sus torres cada vez más alto. (Neurath 1987 [1932], p. 23)

Asimismo, Hahn, Neurath y Carnap afirman:

Los representantes de la concepción científica del mundo están resueltamente de pie sobre el terreno de la simple experiencia humana. Confiadamente ponen manos a la obra en la tarea de remover los escombros

Revista de Filosofía Diánoia, vol. 64, no. 83 (noviembre de 2019-abril de 2020) e-ISSN: 1870-4913 • DOI: https://doi.org/10.22201/iifs.18704913e.2019.83.1717 
milenarios de la metafísica y la teología. (Hahn, Neurath y Carnap 2002 (1929), p. 123)

En el mismo texto leemos:

El aumento de inclinaciones metafísicas y teologizantes que se manifiesta hoy en muchas uniones y sectas, libros y revistas, en conversaciones y clases universitarias, parece estar basado en las feroces luchas sociales y económicas del presente: un grupo de combatientes, aferrándose a formas sociales del pasado, también cultiva las posiciones de la metafísica y de la teología heredadas, a menudo largamente superadas en el contenido, mientras que el otro grupo, vuelto hacia los nuevos tiempos, especialmente en Europa central, rechaza esas posiciones y hace pie en el suelo de la ciencia de la experiencia. (Hahn, Neurath y Carnap 2002 [1929], p. 123)

La interpretación de Horkheimer advierte que no se puede realizar debidamente la adopción de una posición antimetafísica, ya que la negación de determinadas esencias conlleva necesariamente la afirmación de otras. Sin embargo, en su argumentación deja de lado la connotación política de la posición antimetafísica del positivismo y no la considera en relación con su intención de revertir el orden establecido por los sectores sociales que representan los ideales "metafísicos" y "teologizantes".

iv) Frente a la neutralidad valorativa que se atribuye al positivismo, la visión de la Escuela de Fráncfort coloca como fines de la actividad científica la toma de conciencia de la situación de opresión social y el ideal de emancipación y de reversión de la injusticia social.

Es innegable que tanto el positivismo como el neopositivismo sostuvieron claros compromisos valorativos y políticos ligados a la actividad científica (y también a la filosófica). Así, Comte señala una propiedad fundamental de la filosofía positiva: "que ella puede ser considerada como la única base sólida de la reorganización social, para terminar con el estado de crisis en que se encuentran desde hace tiempo las naciones más civilizadas" (Comte 1980 [1930], p. 45). Incluso dedica la última parte de su Curso de filosofía positiva al desarrollo de esa cualidad.

Por su parte, el tópico del ideal de una ciencia unificada no fue un tema más en el desarrollo del positivismo lógico, sino que desde sus comienzos constituyó un tema planteado de manera expresa en los postulados y en la agenda de discusiones del movimiento. Como parte de la

Revista de Filosofía Diánoia, vol. 64, no. 83 (noviembre de 2019-abril de 2020) e-ISSN: 1870-4913 • DOI: https://doi.org/10.22201/iifs.18704913e.2019.83.1717 
labor conjunta de los positivistas lógicos, tuvo lugar la creación de la enciclopedia de la ciencia unificada. El fundador más comprometido con la colección Einheitswissenschaft fue Neurath, quien repartía su colaboración entre estos volúmenes y Erkenntnis, y quien también participó en la organización de los congresos de la ciencia unificada, junto con Carnap y Morris (1935-1939). El órgano de publicación del proyecto de la ciencia unificada fue la International Encyclopedia of Unified Science.

El movimiento promovió la tarea de unificar y coordinar las ciencias de modo que se pudieran utilizar de manera más adecuada como herramientas para la formación y la planificación deliberada de la vida moderna, con el fin de que se cultivara la actividad científica y epistemológica aun entre los ciudadanos comunes. De este modo, se buscaba planificar mejor una futura ciencia unificada que contribuiría con los objetivos colectivos de la sociedad, en contraste con los autores anticientíficos y reaccionarios, a quienes identificaban como practicantes de una retórica oscurantista.

v) Desde el punto de vista de la teoría crítica, el positivismo lógico es la coronación del ideal baconiano, que concibe la ciencia como dominio y control explicativo y predictivo. La labor de la ciencia social orientada por la racionalidad crítica se define, en contraposición a la concepción positivista (racionalidad instrumental), como una teoría orientada a develar los mecanismos de dominación, sus supuestos y sus procesos ideológicos.

Para analizar el papel que se asigna a la ciencia y a la filosofía en esta oposición, es indispensable tener en cuenta que los conceptos de "filosofía" y de "ciencia" que utilizan ambas posiciones son incompatibles (Barck 2011, p. 35). También hacen esta advertencia Araujo y Medina: "El eje para comprender aquí las críticas de Horkheimer es que, cuando ellos hablan de filosofía, no hablan de lo mismo" (Araujo y Medina 2014, p. 121).

La imagen de la ciencia en la visión positivista que reconstruye Horkheimer en los textos que hemos analizado se liga fuertemente al ideal explicativo y predictivo, el cual caracteriza sobre todo la imagen de la ciencia natural en los textos de Carnap. Sin embargo, no es la imagen que puede desprenderse del enfoque de Neurath, quien sostiene:

Este enfoque es fundamentalmente opuesto a cualquier concepción de la noción de sistema como límite de la investigación científica. Y parece natural y consecuencia inmediata de esta perspectiva la idea de que una "ciencia unificada" sea realizada en una enciclopedia como "modelo" de

Revista de Filosofía Diánoia, vol. 64, no. 83 (noviembre de 2019-abril de 2020) e-ISSN: 1870-4913 • DOI: https://doi.org/10.22201/iifs.18704913e.2019.83.1717 
nuestro conocimiento, ya que no podemos comparar las ciencias históricamente dadas con "la ciencia real"; lo más que podemos alcanzar en nuestro trabajo científico parece ser que es una enciclopedia, construida cooperativamente por los científicos interesados en el empirismo científico. Este programa podría ser llamado "enciclopedismo". (Neurath 2011 [1937], pp. 276-277)

El modelo de Neurath de la ciencia unificada se enmarca en el giro sociológico de su perspectiva, en su convencionalismo y en el pluralismo metodológico que defiende; asimismo, se basa en una concepción que, si se compara con la visión de Carnap, resulta bastante menos fundacionista que el resto de las posiciones del Círculo de Viena.

Por último, el papel de la filosofía es claramente diferenciable en la perspectiva del positivismo lógico y en la de la Escuela de Fráncfort. Para el primero, la filosofía no puede ser una forma de conocimiento autónoma de la ciencia, ya que su labor central es ser una metateoría de la ciencia, elucidar sus conceptos, reconstruir los enunciados, buscar la unidad metodológica y contribuir al desarrollo técnico y social en general. En contraposición, los de Fráncfort sí postulan la autonomía de la filosofía y reivindican su relación con las tradiciones kantiana y hegeliana. En este marco, la tarea crítica — como crítica general a las condiciones de posibilidad del conocimiento y de su justificación- resulta central. La concepción marxista de crítica, reconceptualizada en la tradición de la Escuela de Fráncfort, también será central a la hora de identificar el papel de la filosofía, ya que en ella mantiene su relevancia el ideal de toma de conciencia de los sujetos para lograr la transformación social. En ese sentido, cabe destacar que Horkheimer le asigna un papel importante a la filosofía en el contexto de la investigación científica, ya que a ella le corresponderá actuar como órgano crítico de las ciencias especiales.

Con todo, al estar anclado en esa perspectiva, Horkheimer no logró comprender la de sus adversarios. Según O’Neill y Uebel está claro que "Horkheimer no entendió (y mucho menos apreció) que la concepción de la ciencia unificada de los empiristas lógicos podría, y que la de Neurath evidentemente logró, contener en sí misma una metateoría de la ciencia bajo la forma de una lógica y una pragmática científica" (O’Neill y Uebel 2004, p. 89). El autor no alcanza a captar que la tarea filosófica del positivismo no radica sólo en la reconstrucción y la unificación de las ciencias en el camino de una tarea meramente teórica, sino con miras a una dimensión práctica: lograr una comunicación más eficiente entre los científicos, un entendimiento de los sujetos - científicos o 
no- en las situaciones prácticas de la vida moderna (uno de los objetivos centrales del lenguaje Isotype de Neurath), un crecimiento de la educación científica y una mejora de las condiciones de vida en las grandes ciudades.

Si se pone atención a la cantidad de autores y obras citados por Horkheimer (sobre todo en Horkheimer 2002 [1937]) resulta improbable que el sesgo de su interpretación se deba a un conocimiento poco profundo de las ideas del positivismo, de manera que su crítica parece tratarse de un gesto estratégico con el fin de delinear su propia concepción teórica. Las generalizaciones que hace Horkheimer han repercutido fuertemente en las interpretaciones estandarizadas del positivismo del siglo xx y han contribuido a que se desdibujen algunos aspectos en los que ambas posiciones coinciden: por un lado, en la importancia de la aplicación de los métodos empíricos en la investigación social (que en el caso de la Escuela de Fráncfort deben, a su vez, someterse a la regulación de la racionalidad crítica) y, por otro, en el potencial políticamente transformador de la teoría científica, como muchos autores han demostrado para los enfoques positivistas contemporáneos (Reisch 2009, Stadler 2010, Uebel 1996b, Uebel 2005 y Uebel 2010).

\section{BIBLIOGRAFÍA}

Araujo, C.I. y C. Medina, 2014, "Acerca de la polémica Neurath-Horkheimer: ciencia y política", Diánoia, vol. 59, no. 72, pp. 113-129.

Ayer, A.J., 1958, Lenguaje, verdad y lógica, trad. M. Suárez, Martínez Roca, Barcelona [1a. ed.: 1935].

— 1965, El positivismo lógico, trad. L. Aldama, U. Frisco, C.N. Molina, F.M. Torner y R. Ruiz Harrel, Fondo de Cultura Económica, Madrid [1a. ed.: 1959].

Barck, K., 2011, "The Neurath-Horkheimer Controversy Reconsidered: Otto Neurath's Erwiderung to Max Horkheimer's Attack against the Vienna Circle", en Symons, Pombo y Torres 2011, pp. 31-40.

Carnap, R., 1988, La construcción lógica del mundo, trad. L. Mues de Schrenk, Instituto de Investigaciones Filosóficas-UnAM, México [1a. ed.: 1928].

— 2000, The Logical Syntax of Language, trad. A. Smeaton, Routledge, Londres [1a. ed.: 1937].

Cartwright, N., J. Cat, L. Fleck y T.E. Uebel, 1996, Otto Neurath: Philosophy between Science and Politics, Cambridge University Press, Cambridge.

Cirera, R., A. Ibarra y T. Mormann (comps.), 1996, El programa de Carnap: ciencia, lenguaje y filosofía, Ediciones del Bronce, Barcelona.

Coffa, A., 1991, The Semantic Tradition from Kant to Carnap: To the Vienna Station, Cambridge University Press, Cambridge.

Revista de Filosofía Diánoia, vol. 64, no. 83 (noviembre de 2019-abril de 2020) e-ISSN: 1870-4913 • DOI: https://doi.org/10.22201/iifs.18704913e.2019.83.1717 
Comte, A., 1980, Curso de filosofía positiva, trad. J.M. Revuelta, Orbis Hyspamérica, Barcelona [1a. ed.: 1830].

Friedman, M., 1999, Reconsidering Logical Positivism, Cambridge University Press, Cambridge.

Hahn, H., O. Neurath y R. Carnap, 2002, "La concepción científica del mundo: el Círculo de Viena”, trad. P. Lorenzano, Redes, vol. 9, no. 18, pp. 103-150 [1a. ed.: 1929].

Hegselmann, R., 1987, "Introduction", en McGuinnes 1987, pp. ix-xxi.

Horkheimer M., 1985-1996, Gesammelte Schriften in neunzehn Bänden, Fischer, Fráncfort del Meno, tomo 16.

——, 2000, Teoría tradicional y teoría crítica, trad. J. Muñoz, Paidós, Barcelona [1a. ed.: 1937].

— 2002 , “The Latest Attack on Metaphysics", Critical Theory. Selected Essays, trad. M.J. O’Connell, Continuum, Nueva York, pp. 132-187 [1a. ed.: 1937].

—_ 2015, "La situación actual de la filosofía social y las tareas de un instituto de investigación social”, trad. S. Castro, Cuadernos de Filosofía Latinoamericana, vol. 36, no. 113, pp. 211-224 [1a. ed.: 1931].

Ibarra, A. y T. Mormann, 2003, "Engaged Scientific Philosophy in the Vienna Circle: The Case of Otto Neurath", Technology in Society, vol. 25, pp. 235247.

Leyva, G. y M. Mesquita Sampaio de Madureira, 2012, "Teoría crítica: el indisoluble vínculo entre la teoría social y la crítica normativa", en G. Leyva y E. de la Garza Toledo (comps.), Tratado de metodología de las ciencias sociales: perspectivas actuales, Fondo de Cultura Económica, México, pp. 267-324.

McGuinness, B. (comp.), 1987, Unified Science: The Vienna Circle Monograph Series Originally Edited by Otto Neurath, Now in an English Edition, trad. H. Kaal, Reidel, Norwell, Massachusetts.

Mormann, T., 1996, "El lenguaje en Neurath y Carnap", en Cirera, Ibarra y Mormann 1996, pp. 215-241.

Neurath, O., 1987, "Unified Science and Psychology", en McGuinness 1987, pp. 1-23 [1a. ed.: 1932].

$\longrightarrow$, 2011, "Unity of Science and Logical Empiricism: A Reply", en Symons, Pombo y Torres 2011, pp. 15-30 [1a. ed.: 1937].

O’Neill, J., y T.E. Uebel, 2004, "Horkheimer and Neurath: Restarting a Disrupted Debate", European Journal of Philosophy, vol. 12, no. 1, pp. 75-105.

Prono, M.I., 2010a, "Otto Neurath: relevancia y actualidad de su concepción pluralista de la racionalidad", Tópicos, vols. 19-20, pp. 83-100.

—, $2010 b$, "Enunciados protocolares: la propuesta de Neurath a la luz de reconstrucciones actuales", en III Congreso Iberoamericano de Filosofía de la Ciencia y la Tecnología, Universidad Nacional de Tres de Febrero, Buenos Aires, inédito.

Reisch, G., 2009, Cómo la Guerra Fría transformó la filosofía de la ciencia. Hacia las heladas laderas de la lógica, trad. D. Blanco, Universidad Nacional de Quilmes, Bernal. 
Richardson, A. y T.E. Uebel (comps.), 2007, The Cambridge Companion to Logical Empiricism, Cambridge University Press, Cambridge.

Stadler, F. (comp.), 2003, The Vienna Circle and Logical Empiricism. Re-Evaluation and Future Perspectives, Kluwer, Dordrecht.

—_, 2010, El Círculo de Viena. Empirismo lógico, ciencia, cultura y política, trad. L.F. Segura Martínez, Fondo de Cultura Económica, Santiago de Chile.

Symons, J., O. Pombo y J.M. Torres (comps.), 2011, Otto Neurath and the Unity of Science, Springer, Dordrecht.

Uebel, T.E., 1992, Overcoming Logical Positivism from Within. The Emergence of Neurath's Naturalism in the Vienna Circle's Protocol Sentence Debate, Rodopi, Ámsterdam.

_ 1993 , "Neurath's Protocol Statements: A Naturalistic Theory of Data and Pragmatic Theory of Theory Acceptance", Philosophy of Science, vol. 60, no. 4, pp. 587-607.

—_, 1996a, "On Neurath's Boat", en Cartwright, Cat, Fleck y Uebel 1996, pp. 89-166.

- 1996b, "Anti-Foundationalism and the Vienna Circle's Revolution in Philosophy", The British Journal for the Philosophy of Science, vol. 47, no. 3, pp. 415-440.

- 2005, "Political Philosophy of Science in Logical Empiricism: The Left Vienna Circle", Studies in History and Philosophy of Science, vol. 36, no. 4, pp. 754-773.

, 2009, "Neurath's Protocol Statements Revisited: Sketch of a Theory of Scientific Testimony", Studies in History and Philosophy of Science, vol. 40, no. 1 , pp. 4-13.

— 2010 , "What's Right about Carnap, Neurath and the Left Vienna Circle Thesis: A Refutation", Studies in History and Philosophy of Science, vol. 41, no. 2, pp. 214-221.

Wiggershaus, R., 2010, La Escuela de Fráncfort, trad. M. Romano Hassán, Fondo de Cultura Económica, México [1a. ed.: 1984].

Recibido el 3 de mayo de 2018; revisado el 16 de enero de 2019; aceptado el 24 de abril de 2019. 